\section{AL-AZHAR Dental Journal}

F o r

G i r
The Official Publication of The Faculty of Dental Medicine For Girls, Al-Azhar University Cairo, Egypt.

Print ISSN 2537-0308 • Online ISSN 2537-0316

ADJ-for Girls, Vol. 8, No. 1, January (2021) — PP. 19:26

\title{
The Effect of Mesoporous Silica Nanoparticles Desensitizing Slurry Alone or Combined with Calcium Phosphate on Dentin Permeability (An in Scanning Electron Microscope study)
}

\author{
Basant M. Abdel Samie ${ }^{1 *}$, Mohamed A. Al-Yasaki ${ }^{2}$, Nevin A. Gad ${ }^{3}$
}

\begin{tabular}{|l|} 
Codex : 03/21.01 \\
azhardentj@azhar.edu.eg \\
http://adjg.journals.ekb.eg \\
\hline DOI: $10.21608 /$ adjg.2021.14176.1183 \\
\hline $\begin{array}{l}\text { Restorative Dentistry } \\
\text { (Removable Prosthodontics, Fixed } \\
\text { Prosthodontics, Endodontics, Dental } \\
\text { Biomaterials, Operative Dentistry) }\end{array}$ \\
\hline
\end{tabular}

\section{KEYWORDS}

Desensitizing dentin,

Mesoporous silica,

Hydroxyapatite, nanoparticles.

\begin{abstract}
Purpose: Determining the dentinal tubules occlusion and permeability of desensitized dentin after its treatment with (MSN) slurry alone or combined with calcium phosphate $\left(\mathrm{Ca}^{2} \mathrm{po}_{4} @ \mathrm{MSN}\right)$ versus (NHAp) suspension. Materials and Methods: eighty sound posterior teeth were extracted for orthodontic purpose. Dentin disks of $1 \mathrm{~mm}$ thickness were cut perpendicular to the long axis of the teeth at level of $1 \mathrm{~mm}$ above the cemento-enamel junction. All samples were treated with $6 \%$ citric acid solution for 2 minutes to stimulate sensitive teeth. The samples were divided into four main groups (30 each) according to the material used either $\mathrm{Ca}^{+2}$ and $\mathrm{PO}_{4} @$ MSN slurry, slurry of MSN nanoparticles, NHAp suspension and no treatments a control group. These four groups were further subdivided into two groups according to time to after 1 day and after 4 weeks. In random samples from each group, the samples were evaluated for dentinal tubules occlusion using Environmental Scanning Electron Microscope and for dentin permeability. The samples were photographed using Stereomicroscope. Image analysis software was used for indicating degree of occlusion and the depth of dye penetration. The data were analyzed using two-way ANOVA and Turkey's HSD test. The significance level was set at $\mathrm{p} \leq 0.05$. Results: There was significant difference observed between $\mathrm{Ca}^{2} \mathrm{Po}_{4} @ \mathrm{MSN}$ nanoparticles and NHAp compared to (MSN alone) group but there was insignificant difference between groups $\left(\mathrm{Ca}^{2} \mathrm{Po}_{4} @ \mathrm{MSN}\right)$ and (NHAp) in dentinal tubules occlusion and permeability. Conclusion: $\left(\mathrm{Ca}^{2} \mathrm{Po}_{4} @ \mathrm{MSN}\right)$, MSN alone and NHAp are valuable tools to enhance dentinal tubules occlusion and permeability of desensitizing dentin.
\end{abstract}

- Paper extracted from master thesis titled "The Effect of Mesoporous Silica Nanoparticles Desensitizing Slurry Alone or Combined with Calcium Phosphate on Dentin Permeability".

1. Master degree Candidate at Operative Dentistry Department, Faculty of Dental Medicine for Girls, Al-Azhar University, Cairo, Egypt.

2. Professor of Operative Dentistry, Dean of Faculty of Dental Medicine Sinai University, Egypt.

3. Assistant Professor of Operative Dentistry, Faculty of Dental Medicine For Girls. Al-Azhar University, Cairo, Egypt.

* Corresponding author email: mogiegy@gmail.com 


\section{INTRODUCTION}

Dentin hypersensitivity is a disease resulting from dentin surface exposure(1). The hydrodynamic theory of dentin hypersensitivity explains that the fluid moves inside or outside the dentinal tubules in response to stimulus leading to dentin hypersensitivity ${ }^{(2)}$. According to this theory, there are two common processes for the treatment of dentin hypersensitivity: desensitization of the nerve or dentinal tubules occlusion. The dentinal tubules occlusion would probably show more effectiveness in dentin hypersensitivity treatment.

Artificial silica-based products have a successful role in dentinal tubules occlusion ${ }^{(3)}$. The reason for silica ability to occluding dentinal tubules may be due to their hydroxyl groups which have the ability to bind silica to the calcium receptors on the dentin surface $^{(4)}$. Nanomaterials are one of most superior materials for dentinal tubule occlusion because of its superior dispersion of entering dentinal tubules easily about 2-3 mm diameters. The nanoparticles properties are completely dissimilar those of their equivalent bulk materials. The nanoparticles solubility and reactivity are significantly increased because of their large surface area and high surface energy which provides easily deposition on irregular spaces ${ }^{(5)}$.

When MSNs are used for dentinal tubules occlusion, Nano-sized MSNs with superior dispersion, high solubility and reactivity would easily enter the dentinal tubules of 2-3 Mm diameters. They could easily adhere to the dentin surface because of their high similarity and the aid of hydroxyl groups on their surfaces. Calcium and phosphates can be encapsulated in MSNs as calcium and phosphates sources. It is prepared by mixing $\mathrm{CaCo} 3$-containing MSN with $30 \% \mathrm{~h}_{3} \mathrm{po}_{4}$. The super saturation of $\mathrm{Ca}^{2}$ and $\mathrm{Po}_{4}$ ions could improve calcium and phosphate precipitations that can enter more deeply in dentinal tubules .They have the ability to improve the longevity of dentinal tubule occlusion due to its slowly releasing of $\mathrm{Ca}^{2}$ and $\mathrm{Po}_{4}$ in tubules ${ }^{(6)}$.
Nanohydroxyapatite (nHAp) has the capacity to facilitate crystal deposition and growth on demineralized teeth ${ }^{(7)}$. It has Calcium and phosphate ions reservoir to maintain the supersaturated state for tooth mineralization. The inorganic phase of dentin includes large amounts of calcium carbonates, zinc, fluoride and magnesium but the Hydroxyapatite is principal in organic component. So, synthetic HA is considered a logical mineral compound to replace the natural mineral constituent of dentin ${ }^{(8)}$. This study aims to investigate the effect of Mesoporous silica nanoparticles alone or combined with calcium and phosphate versus Nanohydroxyapatite slurries on dentinal tubules occlusion of desensitizing dentin using Scanning Electron Microscope to determine degree of dentinal tubules occlusion and Stereomicroscope to determine the length of penetration depth.

\section{MATERIAL AND METHODS}

A total of 120 premolars were used in this study. The 120 samples were be divided into four main groups (30 each) according to the material used either $\mathrm{Ca}^{+2}$ and $\mathrm{PO}_{4} @$ Mesoporous silica slurry $\left(A_{1}\right)$, slurry of Mesoporous silica nanoparticles $\left(A_{2}\right)$,Nanohydroxyapatite(nHAp) suspension $\left(A_{3}\right)$ and no treatment (control group) $\left(\mathrm{A}_{4}\right)$. These four groups further subdivided into two groups according to time: after 1 day (B1) and after 4 weeks (B2). In SEM 80 sound premolars, dentin disks of $1 \mathrm{~mm}$ thickness were cut perpendicular to the long axis of the teeth at level of $1 \mathrm{~mm}$ above the cementoenamel junction using double faced diamond disc in cutting machine. All samples were treated with $6 \%$ citric acid solution for 2 minutes to stimulate sensitive teeth and subjected to ultrasonic cleaning (100B-HB ultrasonic processor, 25000 revolutions per minute) with distilled water for 30 seconds $^{(9)}$. In another 40 sound premolars, cervical cavities were made. Each tooth was sectioned longitudinally, in mesiodistal direction, into two parts (buccal and lingual) using double faced diamond disc (BesQualDia-Disc NY 11373, USA size: S-22mm) in cutting machine (DEMCO, Dental maintenance CO, Bonsall, Calf. U.S.A, Model E96), then each 
tooth part was embedded in heavy body rubber base blocks (BMS 135, BMS Dental s.r.l. via M Buonarroti, Capannoli, Italy) to allow for easy handling of the sample during the application procedures and removal after the dye application procedure. The samples were placed on their pulpal side leaving only the buccal or lingual surfaces exposed ${ }^{(10)}$. In group $\left(\mathrm{A}_{1}\right): \mathrm{Ca}^{+2} @ \mathrm{MSN}$ and $\mathrm{PO}_{4}{ }^{3} @$ MSN powders were mixed with distilled water in a ratio of $\mathrm{Ca}^{2+} @ \mathrm{MSN}: \mathrm{Po}_{4}{ }^{3-} @ \mathrm{MSN} ; \mathrm{H}_{2} \mathrm{O}=0.015 \mathrm{~g}$ : $0.015 \mathrm{~g}: 150 \mathrm{~mL}$ to make the desensitizing slurry. In group $\left(\mathrm{A}_{2}\right)$ : Mesoporous silica nanoparticles slurry was prepared under identical conditions with a constant TEOS/ Surfactant molar ratio of 10:1. MSNs powder which was mixed with distilled water at ratio of MSNs: $\mathrm{H}_{2} \mathrm{O}=0.015 \mathrm{~g}$ : $75 \mu \mathrm{L}$ to make the desensitizing slurry ${ }^{(9)}$. In group $\left(\mathrm{A}_{3}\right): 1.8$ $\mathrm{g}$ of nHAp was made into slurry by mixing with $0.3 \mathrm{~mL}$ of distilled water ${ }^{(11)}$. In group $\left(\mathrm{A}_{4}\right)$ : Citric acid etched samples without any treatment ${ }^{(9)}$. The desensitizing slurry was immediately applied on the dentin samples with a dental brush. After $30 \mathrm{sec}$, the slurry was applied again. The specimens were stored in artificial saliva solution either for one day or four weeks in labeled sealed containers at room temperature.

\section{Scanning Electron Microscope:}

The surfaces of selected samples were examined using Scanning Electron Microscope (ESM) for evaluating the changes in surface topography; the specimens were dried and mounted on metal stubs; imaged from the selected sample were obtained at $6000 \times$ magnification.

\section{Dentin permeability assessment:}

Dentin permeability test was done using dye penetration method to evaluate the occluding capacity of each treatment modality. Five percent (5\%) by weight Evans blue solution was employed to analyze the dentin permeability. Each sample was sectioned longitudinally in bucco-lingual direction (through the cervical cavity) using double faced diamond disc in cutting machine. The samples were photographed by Steromicroscopy at 40 magni- fication and images of 5.1 megapixels by digital camera were obtained. Three measurements (upper, middle and lower region in the analyzed area) were taken for each image, indicating the depth of the dye infiltration (length of dye penetration), and the mean was calculated for each specimen. The same examiner performed all of the measurements ${ }^{(10)}$.

\section{Statistical Analysis:}

All the data of SEM and dentin permeability results were collected, tabulated and analyzed. One way Anova followed by Pair- wise Tukey's posthoe tests were performed to detect significance between groups. Student t-test was done between material subgroups. Two-Factorial AA was done to detect effect of Variables affecting mean value (experimental groups and time). Statistical analysis was performed using Graph-Pad Instate statistics software for Windows (www. graphpad.com).

\section{RESULTS}

\section{SEM Results:}

The mean values and standard deviation of all experimental groups as a function of evaluation time were summarized in table (1), where in group (A1): when $\mathrm{Ca}^{+2}$ and $\mathrm{Po}_{4} @$ Mesoporous silica slurry was added to the demineralized dentin and the specimens were immersed in artificial saliva for one day recorded a mean value of $(97.71 \pm 0.37)$ and when the specimens immersed in artificial saliva for four weeks recorded a mean value of (98.32 \pm 0.79$)$. Group (A2); when MSN slurry was added to the demineralized dentin and the specimens were immersed in artificial saliva for one day recorded a mean value of $(92.71 \pm 1.42)$ and when the specimens immersed in artificial saliva for four weeks recorded a mean value of (95.96 \pm 1.30). Group (A3); when NHAp slurry was added to the demineralized dentin and the specimens were immersed in artificial saliva for one day recorded a mean value of $(97.29 \pm 0.41)$ and when the specimens immersed in artificial saliva for four weeks recorded a mean value of $(97.58 \pm 0.39)$. Group (A4); when $6 \%$ citric acid solution was added to the dentin and 
the specimens were immersed in artificial saliva for one day recorded a mean value of $(14.95 \pm 0.94)$ and when the specimens immersed in artificial saliva for four weeks recorded a mean value of $(16.65 \pm 0.56)$. It is shown that $\mathrm{Ca}^{+2}$ and $\mathrm{Po}_{4} @$ Mesoporous silica slurry at both evaluation time exhibited the highest mean value group (A1) followed by NHAp slurry group (A3) and followed by MSN slurry group (A2). While $6 \%$ citric acid solution exhibited the lowest mean value group (A4).

Table (1): The mean, standard deviation (SD) values of degree of occlusion of different groups

\begin{tabular}{|c|c|c|c|c|c|}
\hline \multirow{3}{*}{ Variables } & \multicolumn{5}{|c|}{ Total area of closed dentinal tubules $(\%)$} \\
\hline & \multicolumn{2}{|c|}{$\begin{array}{l}\text { After } 1 \text { day } \\
\text { (B1) }\end{array}$} & \multicolumn{2}{|c|}{$\begin{array}{l}\text { After } 4 \text { weeks } \\
\quad \text { (B2) }\end{array}$} & \multirow[t]{2}{*}{ p-value } \\
\hline & Mean & SD & Mean & SD & \\
\hline $\begin{array}{l}\text { CaPO4@ } \\
\text { msn (A1) }\end{array}$ & $97.71^{\mathrm{aA}}$ & 0.37 & $98.32^{\mathrm{aA}}$ & 0.79 & $0.061 \mathrm{~ns}$ \\
\hline MSN (A2) & $92.71^{\mathrm{bB}}$ & 1.42 & $95.96^{\mathrm{bA}}$ & 1.30 & $0.023 *$ \\
\hline NHAP (A3) & $97.29^{\mathrm{aA}}$ & 0.41 & $97.58^{\mathrm{aA}}$ & 0.39 & $0.357 \mathrm{~ns}$ \\
\hline Control (A4) & $14.95^{\mathrm{cB}}$ & 0.94 & $16.65^{\mathrm{cA}}$ & 0.56 & $0.030 *$ \\
\hline p-value & \multicolumn{2}{|c|}{$<0.001 *$} & \multicolumn{2}{|c|}{$<0.001 *$} & \\
\hline
\end{tabular}

Means with different small letters in the same column indicate significant difference, Means with different capital letters in the same row indicate significant difference.*; significant $(p<0.05)$ ns; non-significant $(p>0.05)$

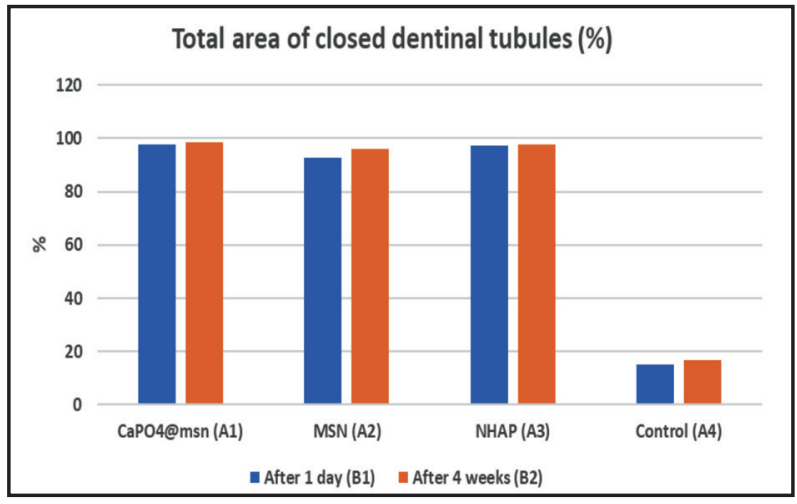

Figure (1): Bar chart representing degree of occlusion for different groups: $\mathrm{Ca}{ }^{2} \mathrm{Po}_{4} @ \mathrm{MSN}(\mathrm{A} 1), \mathrm{MSN}$ (A2), NHAp(A3) and Citric acid control group (A4) comparing between after one day and after four weeks: $\mathrm{B} 1$ and $\mathrm{B} 2$.

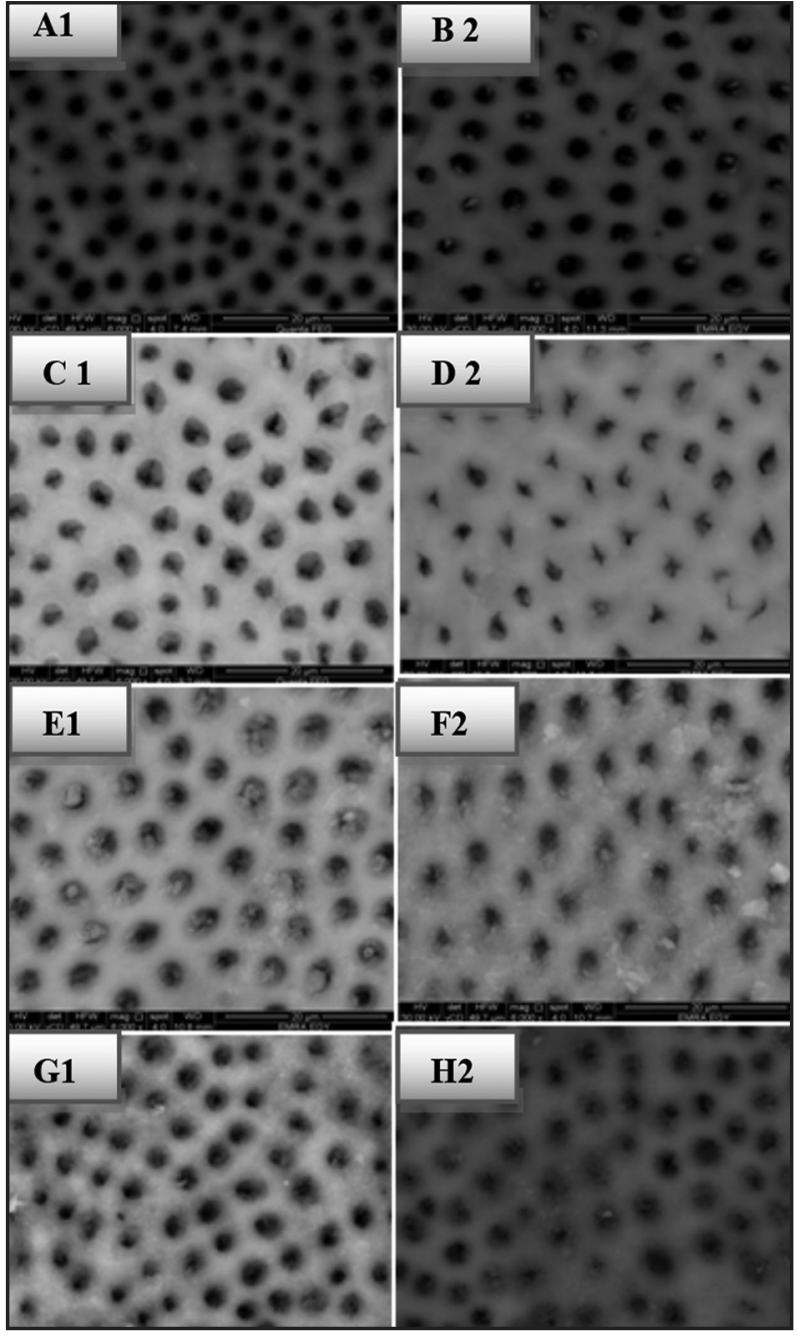

Figure (2): SEM images of surface morphological changes of dentinal tubules: A1: referring to Citric acid treated sample after one day, B1: referring to Citric acid treated sample after 4 weeks, C1: referring to $\mathrm{Ca} 2 \mathrm{Po} 4 @$ MSN treated sample after one day, D2: referring to Ca 2Po4@MSN treated sample after four weeks.E1: referring to MSN treated sample after one day, F2: referring to MSN treated sample after four weeks, G1: referring to NHAp treated sample after one day, $\mathrm{H} 2$ : referring to NHAp treated sample after four weeks.

\section{Stereomicroscope Results:}

The mean values and standard deviation of all experimental groups as a function of evaluation time were summarized in table (2). The highest mean value was found after one day in control group(630.06 \pm 96.67$)$ followed by (MSN)group

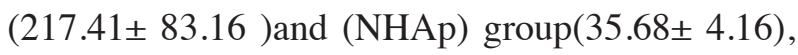


while the least mean value was found in $\left(\mathrm{Ca}^{2} \mathrm{Po}_{4}\right)$ group(33.01 \pm 2.69$)$. The highest mean value was found after 4 weeks in control group (514.27士 71.93) followed by ( MSN ) group (129.26 \pm 43.60$)$ and (NHAp) group $(31.03 \pm 4.03)$, while the least mean value was found in $\left(\mathrm{Ca}^{2} \mathrm{Po}_{4} @ \mathrm{MSN}\right)$ group.

Table (2): The mean, standard deviation (SD) values of dye penetration of different groups

\begin{tabular}{|c|c|c|c|c|c|}
\hline \multirow{3}{*}{ Variables } & \multicolumn{5}{|c|}{ Dye penetration } \\
\hline & \multicolumn{2}{|c|}{$\begin{array}{l}\text { After } 1 \text { day } \\
\text { (B1) }\end{array}$} & \multicolumn{2}{|c|}{$\begin{array}{l}\text { After } 4 \text { weeks } \\
\text { (B2) }\end{array}$} & \multirow{2}{*}{ p-value } \\
\hline & Mean & SD & Mean & SD & \\
\hline $\mathrm{Ca}^{2} \mathrm{Po}_{4} @ \mathrm{msn}(\mathrm{A} 1)$ & 33.01 & 2.69 & 27.77 & 2.14 & $0.013^{*}$ \\
\hline MSN (A2) & 217.41 & 83.16 & 129.26 & 43.60 & $0.060 \mathrm{~ns}$ \\
\hline NHAP (A3) & 35.68 & 4.16 & 31.03 & 4.03 & $0.001 *$ \\
\hline Control (A4) & 630.66 & 96.67 & 514.27 & 71.93 & $0.008^{*}$ \\
\hline p-value & \multicolumn{2}{|c|}{$<0.001 *$} & \multicolumn{2}{|c|}{$<0.001 *$} & \\
\hline
\end{tabular}

Means with different small letters in the same column indicate significant difference, Means with different capital letters in the same row indicate significant difference. *; significant $(p<0.05) n s$; non-significant $(p>0.05)$

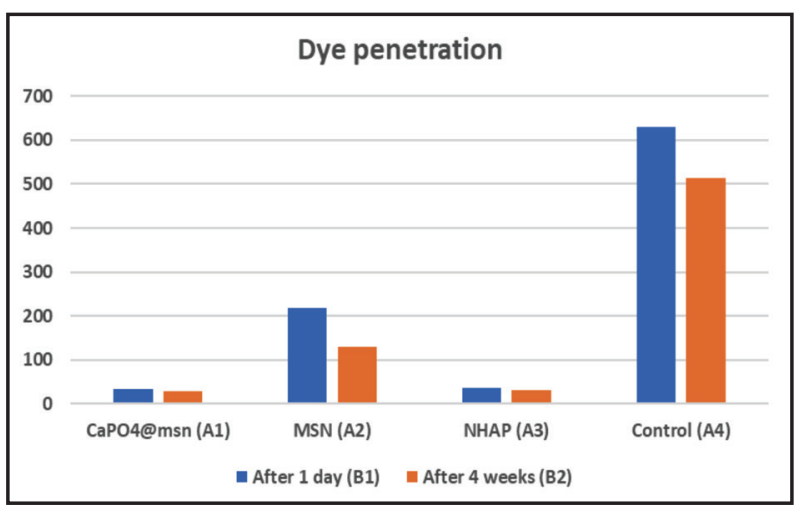

Figure (3): Bar chart showing dye penetration for different groups: A1, A2, A3 and A4 comparing between after one day and after four weeks: B1 and B2.

\section{DISCUSSION}

The present study was done to evaluate the dentinal tubules occlusion and permeability of desensitized dentin after its treatment with Mesoporous silica nanoparticles slurry alone or combined with calcium phosphate versus Nanohydroxyapatite suspension. Dentin is a vital tissue consisting of dentinal tubules which is naturally sensitive because of extensions of odontoblasts and formation of dentine-pulp complex ${ }^{(11)}$. Dentin hypersensitivity is one of the most current diseases in clinic. There are two treatment options for dentin hypersensitivity which are desensitization or dentinal tubules occlusion, the later was proven that it is more effective. Depending on dentin hypersensitivity Hydrodynamic theory, it can be managed by decreasing the dentin permeability through dentinal tubules occlusion which lead to decreasing the fluid flow movement inside the tubules .It was proven that any management for dentin permeability is considered a treatment for dentin hypersensitivity ${ }^{(12)}$.In recent years, many research groups have attempted to desensitize the open dentinal tubules and decreasing the dentin permeability by chemical desensitizing agents such as sodium fluoride, bioactive glasses and silica ${ }^{(13)}$. These desensitizing agents effectively desensitize opened dentinal tubules to some extent superficially with short-lived, limited depth infiltration and their effect diminished during teeth brushing , food chewing, dissolved by saliva or consumption of acidic beverage ${ }^{(14)}$. So Nanomaterials are used nowadays which have the ability to diffuse deeper with more sealing to dentinal tubules more than the conventional bulk materials because of its high surface energy and large surface area but their effect of desensitization still needs to be improved.

120 freshly extracted human premolars were used as a substrate in this study. Specimens prepared from human teeth are preferred because they allow for testing of the study hypothesis in a more clinically relevant substrate. Nevertheless, some variations with the use of human teeth exist, such as the differences in patient's age and the 
environmental factors can lead to subtle differences in enamel characteristics and influence the ability of an acid to properly demineralize the tissues those effects were minimized in this study by including teeth from patient age range of (18-25 years). Since premolars teeth were more available than other teeth due to its extraction for orthodontic treatment, they were enrolled in this study.

Dentin disks were standardized as much as possible with concern with teeth age, teeth positions and dentinal tubules direction. Experimental areas were attentively checked to be sure that they were free of coronal enamel, pulpal exposures, 2ry dentin, or microcracks. Coronal dentin discs were used in this study to evaluate tubule occlusion of the desensitizing agents into the tubules. Important variables such as dentin surface area, thickness and surface characteristics can be controlled in coronal dentin discs compared to cervical dentin discs ${ }^{(11)}$. But for dye penetration test, cervical cavities are made in sound premolars for easily dye injection by $5 \%$ Evans blue dye material for dentin permeability measurements.

Mesoporous silica nanoparticles (MSNs) have been long studied and attracted a lot of attention in the previous studies because of its perfect biocompatibility, large surface area, high surface energy and thermal stability properties. From there, MSN was also proved that it is a perfect medium for nanoparticles carrier in different drug delivery therapies ${ }^{(15)}$. It was demonstrated that calcium and phosphate super saturation minerals which result from mixing of $\mathrm{CaCo}_{3}$ containing MSN with $\mathrm{H}_{3} \mathrm{Po}^{4}$ have the ability of penetrate the dentinal tubules in a very deep extent. Using MSNs and $\mathrm{Ca}^{2} \mathrm{Po}_{4} @ \mathrm{MSNs}$ were improved in the previous studies in their efficiency and accuracy in deposition at both the external dentinal tubules orifices and in the depth of dentinal tubules because of its uniform small size sphere and high order porous structure. MSNs was used in this study as it has been revealed that it has an affinity towards calcium ions, which can act as mineralization reservoir for calcium phosphate minerals that help in dentinal tubules occlusion ${ }^{(13)}$.
Nanohydroxyapatite was used in the present study because it was proven in previous studies that it has potential role in desensitize the dentinal tubules because of its small size, $\mathrm{ca}^{2}$ and $\mathrm{po}_{4}$ attracting ability as a reservoir and crystal growth deposition inside dentinal tubules walls which make a superior dentinal tubules occlusion .Nanohydroxyapatite also have main advantage in dentistry that it could mimic the features of tooth enamel and dentin. It is about $97 \%$ of enamel and about $70 \%$ of dentin ${ }^{17}$. Because of its acceptable cost and the ability of $\mathrm{Ca}$ reservoir, its usage always increases in treating dentin hypersensitivity. Due to the superior bioactive features of Nanohydroxyapatite, it may be the excellent management for dentin hypersensitivity in the near future. The Engineering opinion about NHAp confers superior functional properties to NHAp such as high bioactivity due to its grain size, crystalinity and surface area to volume ratio in the body environment compared to its micro scale sized counterparts. Because of increasing the surface area and atomicity with decreasing micro scales size, a greater penetration of ion constituents into the dentinal tubules would have occurred that might have resulted in better tubular occlusion ${ }^{(18)}$.

Citric acid was used in the present study as it has the ability to open and wide the dentinal tubules with removing the smear layer to allow the desensitizing slurry penetrate the dentinal tubules to their maximum abundant extent ${ }^{(19)}$. It was proven that citric acid under the concentration of $6 \%$ that dentin permeability increased rapidly and after citric acid etching for $30 \mathrm{~s}$, reaching to the maximum depth could be happen ${ }^{(20)}$.

Dentin permeability reduction is affected by coating strategy and the storage medium. Using artificial saliva as a storing medium because it can resample saliva mineral crystals and act as equivalent solution ${ }^{(18)}$. It is helpful in determining the durability of different desensitizing agents under conditions resample the conditions of oral cavity. It was proven that artificial saliva is better than deionized water in vitro studies as storage 
medium. It contains mineral crystals depositions inside the dentinal tubules walls and peritubular dentin resulting in dentin permeability reduction and dentinal tubules constriction. Storing the specimens in it for one day and 4 weeks to compare the effect of different desensitizing slurry in short and long time.

Using Scanning electron microscope (SEM) in the present study as it gives very detailed image of the micomorphological changes in dentin with the help of using Dye penetration test. The latter is a low cost method and a good alternative to test the sealing ability of the different desensitizing slurries.

In the present study, comparing the percentage change of mean values of each group, the results revealed that the highest mean values were recorded for $\mathrm{Ca}^{2} \mathrm{Po}_{4} @ \mathrm{MSN}$ group followed by NHAp group followed by MSN group and citric acid group recorded the lowest mean value. There was statistically significant difference $\mathrm{Ca}^{2} \mathrm{Po}_{4} @ \mathrm{MSNs}$ group and $\mathrm{MSN}$ alone group. $\mathrm{Ca}^{2} \mathrm{Po}_{4} @ \mathrm{MSNs}$ group is higher, it may be because it has more $\mathrm{Ca}^{2}$ and $\mathrm{Po}_{4}$ ions to be released which enhance dentinal tubules occlusion that increasing the ability of rapid initial mechanical sealing and decreasing the dentin permeability ${ }^{(12)}$.It may be also mixing of $\mathrm{CaCo}_{3}$ containing $\mathrm{MSN}$ with $\mathrm{H}_{3} \mathrm{Po}^{4}$ may increase the dentinal tubules penetration in a very deep extent ${ }^{(12)}$.

There was insignificant difference between $\mathrm{Ca}^{2} \mathrm{PO}_{4} @ \mathrm{MSN}$ group and NHAp but $\mathrm{Ca}^{2} \mathrm{Po}_{4} @$ MSNs is higher may be because when MSNs mixed during preparation with distilled water, easily and rapidly penetrating and depositing inside the dentinal walls happened because of its high surface area . It was proven that the slow releasing of $\mathrm{Ca}^{2}$ and $\mathrm{PO}_{4}$ ions of $\mathrm{Ca}^{2} \mathrm{Po}_{4} @ \mathrm{MSN}$ inside the dentinal tubules lead to increasing life time of remineralization process. $\mathrm{Ca}^{2}$ and $\mathrm{Po}_{4}$ ions deposited rapidly and separately without any reaction with etch other inside the tubules leading to initial sealing ${ }^{(12)}$.

There was significant difference between Nanohydroxyapatite (NHAp) group and MSN alone group. NHAp group is higher and that may be because NHAp is osteoconductive material which means that it has the ability to providing Calcium and Phosphate inside the dentinal tubules in a very fast rate but MSNs is osteoinductive material which means that it has the ability to attracting calcium and phosphate in a slow rate. Therefore, the dentinal tubules occlusion is slower in MSN group than NHAp group. It may be also due to the similarity between the HA and the natural apatite within the dentinal tubules. The previous studies proved that the small size, atomic number on their surfaces and very high surface energy with strong combination with other atoms allow it to desensitize dentinal tubules in a fast rate. The acidic $\mathrm{pH}$ of Nanohydroxyapatite may be also a corresponding factor for deeper tubular penetration ${ }^{(18)}$.

Citric acid group recorded the least mean value because almost all the dentinal tubules completely opened to prepare them for receiving the desensitizing slurries ${ }^{(20)}$.

When comparing the change of mean value of each group after one day and after 4 weeks, the result revealed that the highest mean value recorded for 4 weeks because crystal deposition from the artificial saliva inside the dentinal walls was proven that it helps in dentinal tubules occlusion and decreasing the dentin permeability. This difference was statistically insignificant as revealed by Anova test.

SEM observations of the present study correlates well with the Dye penetration test results while in $\mathrm{Ca}^{2} \mathrm{Po}_{4} @ \mathrm{MSN}$ group, the opened dentinal tubules were almost occluded and part of the dentin surface was covered with newly formed precipitates after one week and tubules were completely occluded and the dentin surface was covered with newly formed precipitates with infiltrating to a deeper after four weeks. In Nanohydroxyapatite group, the opened dentinal tubules were partially occluded with newly formed precipitates after one day and almost completely occluded with infiltrating to a deeper after four weeks. In MSN alone group, the 
opened dentinal tubules were partially occluded with newly formed precipitates after one day and were completely occluded while some were almost completely occluded with infiltrating to a deeper after four weeks.

\section{CONCLUSION}

All treatment materials used were effective in dentinal tubules occlusion and dentin permeability; with $\mathrm{Ca}^{2} \mathrm{Po}_{4} @ \mathrm{MSN}$ being the most effective after one week and after four weeks. All the treatment materials produced micro morphological changes of the dentin surface in terms of occluding the orifices of dentinal tubules to variable degrees.

Endnotes

\section{REFERENCES}

1. Absi E, Addy M, Adams D.Dentine hypersensitivity.A study of the patency of dentinal tubules in sensitive and nonsensitive cervical dentine.J Clin Periodontol 1987; 14: 280- 4.

2. Brannstrom M. Dentin sensitivity and aspiration of odontoblasts. J Am Dent Assoc 1963; 66: 366-70.

3. Addy M, Mostafa P, Dentin hypersensitivity produced by the uptake in vitro of toothpastes. Oral Rehabil1989; 16: 35-48.

4. West N, Hughes J. Addy M .Dentin hypersensitivity the effects of brushing toothpaste on etched and unetched dentin in vitro.J oral Rehabil 2002; 29: 167-74.

5. De M. Aramwit P, Kwon G.Nanotechnology in drug delivery. New York: Springer-AAPS Press; 2009; 2; 1-28.

6. Chiang Y, Chen H, Liu H, Kang S, Lee B, Lin F, Lin H. Lin C.A novel Mesoporous biomaterial for treating dentin hypersensitivity. J Dent Res 2010; 89: 236-40

7. Huang S, Gao S, Cheng L, Yu H. Remineralization potential of nano hydroxyapatite on initial enamel lesions: An in vitro study, Caries.Research 2011;45:460-8.

8. Weatherell J. Composition of dental enamel. British Medical Bulletin 1975;31:115

9. Lili T, Ce P, Ying S, Xuan G, Bo Z, Juanjuan Q. Effects of Mesoporous silica nanoparticles upon the function of mammalian sperm in vitro. Dental Materials Journal 2014, $33: 125-32$

10. Mosleh A, Niazy M, El-Yassaky M. Clinical and laboratory evaluation of the efficacy of three Difference modalities in management of dentin hypersensitivity. Al Azhar Dental Journal for Girls 2018; 5: 129-34.

11. Sanjay M, Vivek A, Bhoomika A. Dentin hypersensitivity: Recent trends in management. J conserve Dent 2010; 13:218-24.

12. Davari A, Ataei E, Assarzadeh H. Dentin Hypersensitivity: Etiology, Diagnosis and Treatment; A Literature Review. J Dent 2010; 14:136-45.

13. Lili T, Ce P, Ying S, Xuan G, Bo Z, Juanjuan Q. Effects of Mesoporous silica nanoparticles upon the function of mammalian sperm in vitro. Dental Materials Journal 2014, 33:125-32.

14. Lee B, Tsai H, Tsai Y, Lan w, Lin C.In vitro study of DPbioglass paste for treatment of dentin hypersensitivity. Dent Mater J 2005; 24:562-9.

15. Liong B, France B, Bradely K, Zink J. Antimicrobial activity of silver nanocrystals encapsulated in Mesoporous silica nanocrystals in mesoporous silica nanoparticles. Adv Mater 2009; 21: 1684-9.

16. Jian Y, Hongye Y, Kang L, Jinmei L, Liqun Z, Cui H .A novel application of Nanohydroxyapatite /Mesoporous silica biocomposite on treating dentin hypersensitivity. Journal of Dentistry 2016; 5712: 30067-7.

17. Ohta K, Kawamata H, Ishizaki T, Hayman R. Occlusion of dentinal tubules by nano-hydroxyapatite. J Dent Res.2007; 86:1759.

18. Divya K, Sujatha M, Vidhya S, Mahalaxim S. Evaluation of dentinal tubule occlusion and depth of penetration of nano-hydroxyapatite derived from chicken eggshell powder with and without addition of sodium fluoride. J conserves Dent 2016, 19: 239-44.

19. Khademi A, Feizanfard M.The Effect of EDTA and Citric Acid on Smear Layer Removal of Mesial Canals of First Mandibular Molars. Journal of Research in Medical Sciences 2004; 2: 80-8.

20. Pashley D, Michelich V, Kehl T. Dentin permeability: effects of smear layer removal. J Prosthet Dent 1981; 46: 531. 